\title{
Identifying and Visualizing Relevant Deviations in Longitudinal Sensor Patterns for Care Professionals
}

\author{
Saskia Robben, Mario Boot, Marije Kanis and Ben Kröse \\ Amsterdam University of Applied Sciences \\ Create-IT - Digital Life \\ Duivendrechtsekade 36-38, 1096AH, Amsterdam \\ Email: \{s.m.b.robben, mario.boot, m.kanis, b.j.a.krose\}@hva.nl
}

\begin{abstract}
Sensor technology is increasingly applied for the purpose of monitoring elderly's Activities of Daily Living (ADL), a set of activities used by physicians to benchmark physical and cognitive decline. Visualizing deviations in ADL can help medical specialists and nurses to recognize disease symptoms at an early stage. This paper presents possible visualizations for identifying such deviations. These visualizations have been iteratively explored and developed with three different medical specialists to better understand which deviations are relevant according to the different medical specialisms and explore how these deviations should be best presented. The study results suggest that the participants found a monthly bar graph in which activities are represented by colours as the most suitable from the ones presented. Although the visualizations of every ADL was found to be more or less relevant by the different medical specialists, the preference for focusing on specific ADL's varied from specialist to specialist.
\end{abstract}

\section{INTRODUCTION}

The increasing ageing of the population and the growing world population [1] is expected to result in a surplus of elderly people and a shortage of young people to care for the elderly. Consequently, the need for professional and cost-efficient care is also growing. To address this issue, sensor technology is particularly explored to support successful ageing in place. For this purpose, sensors can be installed in the home for monitoring the activities of elderly people [2]. The data gathered by the sensors can reveal information about their activities and signal physical and cognitive decline. Medical specialists are particularly interested in the daily pattern of activities [3]. Particularly deviations in these daily patterns are useful for the medical specialists, for adapting their care to the specific needs of every patient.

A lot of researchers in the field of pervasive health visualise their sensor data to get a grasp of their data, and to share insights with fellow researchers. This results in interesting visualizations, such as activity density maps [4], average daily pattern graphs [5] or even 3d-representations of sensor data [6]. Additionally, data mining techniques also apply to this field [7]. However, there is also work that particularly target other user groups, such as (in)formal carers or elderly themselves. For example, researchers [8] identified seven types of AAL (Ambient Assisted Living) service users and for each user they identified the contribution of visualizations to decision support. Other researchers developed a system that generates email alarms for care professionals, which uses the feedback of those experts to improve the alarms [9].
This paper describes the development of the visualizations and particularly the identification of relevant deviations through iterative study. It explores desired data visualizations of the sensor output derived from ambient activity monitoring systems. This study includes co-creation sessions with elderly care specialists to gather their opinions and needs with regard to sensor data visualizations. The main questions addressed in the study are: (a) Which deviations in the patterns are relevant for the medical specialists? (b) How should these deviations be represented? Both questions were addressed through feedback gathered in focus group sessions with three medical specialists.

\section{APPROACH}

This work takes place in the context of a Living Lab [10], which means that the care specialists and elderly participants are involved in developing the sensor network and possible sensor output interfaces in real-world settings. More than twenty independently living elderly in our labs have sensor networks installed in their home for long-term monitoring of behaviour. Some reside in assisted living facilities, but others are living in normal neighbourhoods. The sensor networks consist of approximately 16 wireless sensors. For reasons of acceptance and privacy only non-intrusive ambient sensors are used, such as passive infrared motion sensors, magnetic sensors for doors and cabinets, a pressure sensor in the bed and a floating sensor for the toilet. The behaviour of the residents triggers the sensors in sending a sensor event to a local baseunit (data access point). In this unit, the sensor events are gathered and stored in a secured database for further analysis, for example activity recognition or alarm generation.

The resulting database contains a massive amount of data, which is not in a typical comprehensible format for people in the care domain. An example of a basic visualization is shown in Fig. 1. For making efficient use of these data logs, it should be presented in a way so that the data is easy to understand, visually appealing and relevant for the care professionals.

\section{A. Development of Visualizations}

In developing visualizations of the data logs, the main purpose is identifying which specific activity patterns of the residents are indicators of a disease or some other need for professional care. When visualizing ADL's, a regular daily pattern will often emerge. Deviations in these patterns can be signals of an impending disease. Therefore, it is important that the nurses and medical specialists can be aware of such information so they can adapt their treatments. Because medical 


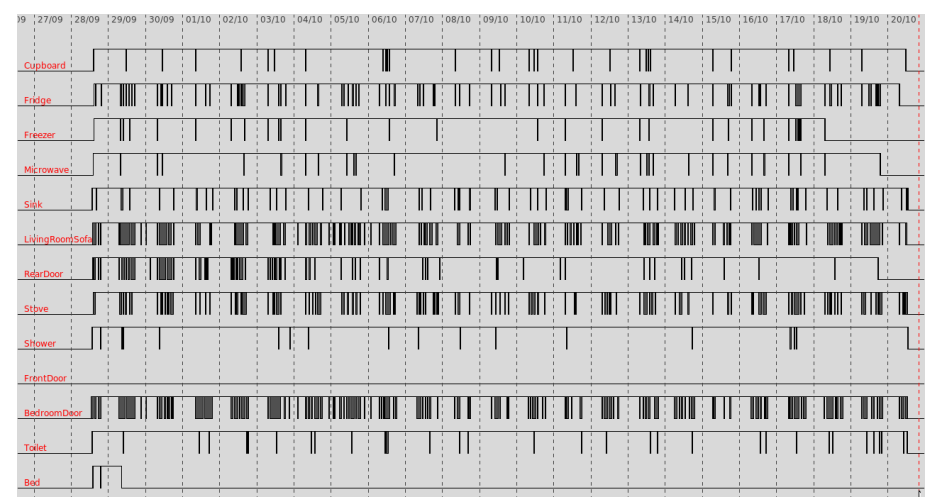

Fig. 1. An example of data visualization tool used by research analysts, $\mathrm{x}$-axis being time and $\mathrm{y}$-axis gives several sensors. Most care professionals will not be able to grasp the meaning of this data.

specialists have limited time available to get informed about one's disease symptoms, relevant deviations in the ADL should pop-out of the screen immediately. For guiding the attention of the specialist to the information that is most relevant for them, knowledge on the laws of gestalt and preattentive variables can help. The laws of Gestalt are about perceiving various small parts as one whole, examples include similarity, continuity and closure [11]. Preattentive variables are grounded in the cognitive process of perception, where some attributes are processed earlier than others. For example the preattentive variables contrast, shadow and border are processed earlier than other variables [12].

Taking these considerations into account different visualizations are constructed for the care specialists. Initially, these were drawn on paper while in a subsequent iteration these are constructed in an application with real data. Before using the visualizations in the focus group sessions, some informal feedback was obtained from an occupational therapist. For providing an insight into daily patterns multiple sketches with different forms of bar charts, line charts and other metaphorlike charts are constructed. An example of visualization in the form of a calendar is given in Fig. 2. Additionally, several ways of displaying deviations of these patterns are developed, including a display with different colours, with a description in words, and with a coloured circle around the deviation.

\section{Study Methods}

The goal of this study is determining (1) which deviations in daily patterns are relevant and (2) how these should be visualized. In developing the visualizations, a participatory design approach was taken. Two focus group sessions with (the same) three medical specialists were organized to address these issues. In addition to the three specialists (physical therapist, occupational therapist and psychologist), attendees also included the director of a care home and an assistant (for note-taking etc). In both sessions leaflets were used in which the prototypes were printed along with questions for each prototype.

\section{A. Session 1}

This two-hour session started with a introduction of sensor monitoring and a discussion of the current workflow of the

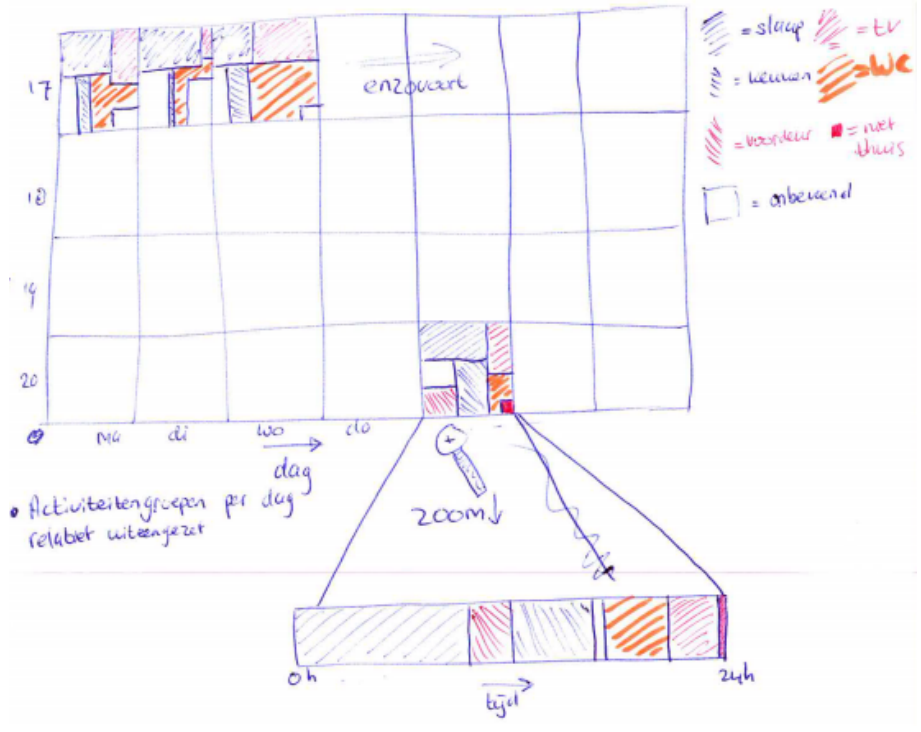

Fig. 2. An example of a visualization prototype used in the first session, where each colour represent the time spent in a location.

specialists. After this, eight paper sketches were used in discussing possible uses of sensor monitoring, examples of what could be relevant changes in patterns en possible ways for visualizing such deviations. In the end, the specialists were asked to choose both the most clear visualization and the visualization that displayed deviations best from all eight visualization examples.

\section{B. Session 2}

This one-hour session started with a short summary of the previous session after which new visualizations, all constructed with real sensor data, were presented and discussed. The previously voted best visualization (a monthly bar pattern) was further developed into 14 versions and three line graphs were added which could provide more details. Discussed were colours and shape, five possibilities to display deviations, what a baseline pattern should look like and zoom possibilities. After this, the question was addressed which deviations in daily patterns are relevant. For identifying relevant deviations in the ADL, the specialists were asked to individually fill in a table which displayed all the ADL's and several other activities which can be measured with the sensor network. The specialists were asked to determine for each activity which one of the three aspects time (what time?), frequency (how often?) and duration (how long?) were relevant to them, and to which extent.

\section{Results AND Discussion}

This study addressed the two questions (1) which deviations in daily patterns are relevant for the medical specialists and (2) how should these deviations be represented. The results are grouped according to these questions.

\section{A. Visualization}

The study participants reported that several aspects are important in the visualizations, which can be divided in four 
TABLE I. P: PSYCHOLOGIST, O: OCCUPATIONAL THERAPIST, F: PHYSIOTHERAPIST. SHOWING WHICH OF THE ACTIVITIES ARE IMPORTANT FOR WHICH OF THE SPECIALISTS (ON AT LEAST ONE OF THE ASPECTS).

\begin{tabular}{l|l|l} 
& \multicolumn{2}{|c}{ Important } \\
\hline ADL/Activity & Yes & No \\
\hline \hline Clothing & P-O-F & \\
Toileting & P-O-F & \\
Washing & P-O-F & \\
Movement & P-O-F & \\
Food preparation & P-O & F \\
Food consumption & P-O & F \\
Sleep & P-O-F & \\
Outside & P-O-F & \\
Sitting in living room & P-F & O \\
Transfers & P-F & O \\
Visits & P-O-F & \\
Nightly activity & P-O-F &
\end{tabular}

categories: (a) the way in which data is represented, (b) a baseline which represents an average pattern, (c) the way deviations are displayed in the data and (d) extra functionalities in the application.

(a) According to the three specialists, long term changes in the activities can be best displayed by showing two months of one-day-intervals under each other. It should be possible to compare multiple activities at the same time, therefore a line chart which displayed the length of one activity per day, was not chosen. Furthermore, it was noted that different activities should be displayed by different colours which correspond to the activities. The colours which were considered best were prime, contrasting colours such as red, blue, green and yellow. The width of the lines should be +-3 millimeter; smaller is considered too small to be clear, bigger is considered too big to display enough time.

(b) The study participants requested a baseline pattern to which they can compare the current activity pattern. This baseline has to represent an average activity pattern. The average should be calculated from the first month of measuring. In case the baseline did change without remarkable deviations, the specialists requested that they should be able to alter the dates between which the baseline is calculated, but the standard setting for the baseline has to be the first month of measured data.

(c) Relevant deviations in the ADL should be signalled with a circle around the deviation, together with an accurate description with the day numbers and red-printed day numbers on the $y$-axis. This combination of a preattentive-based variable and an accurate description provides a double-check. Deviations represented by a slowly changing colour were considered confusing: it was not clear what this colour represented.

(d) With a zoom-selection-tool, the different specialists wanted to be able to zoom and to choose between relevant and irrelevant data (for example show only daytime hours). Furthermore, in order to view the data of multiple residents, a functionality to select different source files is desired.

The final version of the application is shown in Fig. 4. An additional remarkable result was that the specialists identified the person of whom the data was used for creating visualizations, even though the data was anonymously presented and hidden from the session leader.

\section{B. Relevant Deviations}

The different medical specialists had different takes on what they considered relevant deviations in ADL and other

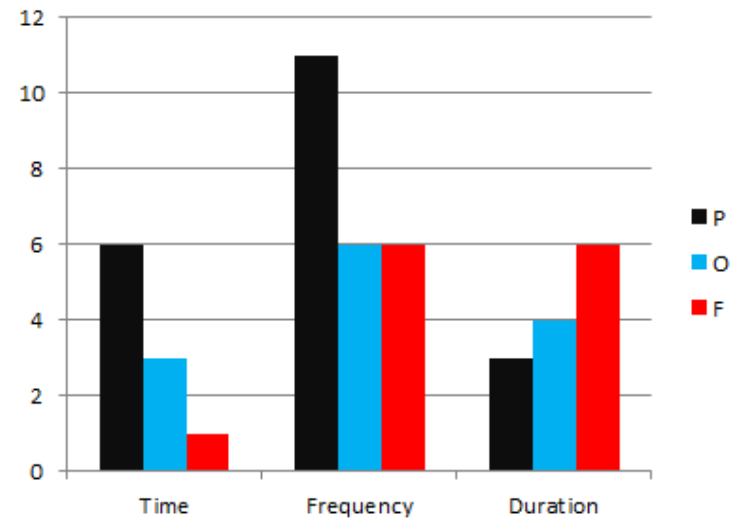

Fig. 3. P: psychologist, O: occupational therapist, F: physiotherapist. Shows how many times each activity-aspect is relevant for each specialist. For example: the $\mathrm{P}$ in the first column means that a change in time is for 6 of 11 activities relevant for the psychologist.

activities. As Table I shows, changes in food consumption and preparation are not important for the physiotherapist, while sitting in the living room and (functional) transfers are not important for the occupational therapist. Three aspects of the activities, frequency, time and duration, were considered of importance and these results are shown in Fig. 3. This figure shows that a change in time of an activity is not very important for the physiotherapist. However, the frequency aspect was considered as very important for the psychologist. Regarding to what extent deviations are relevant, the only thing to be concluded is that the extent of the deviation varies for different specialists.

\section{CONClusion AND Future Work}

Based on the principles of perception and visualization, an application to visualize the sensor data was designed. Input and feedback from medical specialists was used to develop an application which is most suitable to the end-users. The visualizations were also used for discussing and identifying relevant deviations in activities which might lead to more effective care. Different specialists view different aspects of the activities as important. Some activities are not important to all the specialists, but almost all deviations are more or less relevant to each of the specialists.

Regarding the visualizations the specialists stated that they desired extra functionality, such as zooming to view the data in greater or lesser detail, and they want to be able to select relevant times (e.g. view only daytime hours). When such extra functionalities are implemented, care should be taken to evaluate these again with the intended end-users. Another issue related to the visualization is the colours. Though the participants in our study indicated they prefer primary colours, they should also be appropriate for people with colour blindness. Though this study resulted in a useful visualization application for visualizing sensor patterns, and revealed some insights on which deviations might be relevant, it should be noted that only a small number of participants were included in this study. A larger study is needed to provide more valid and reliable conclusions. When designing this study we were not expecting much difference between the several disciplines regarding the relevance of deviations of activities, but the 


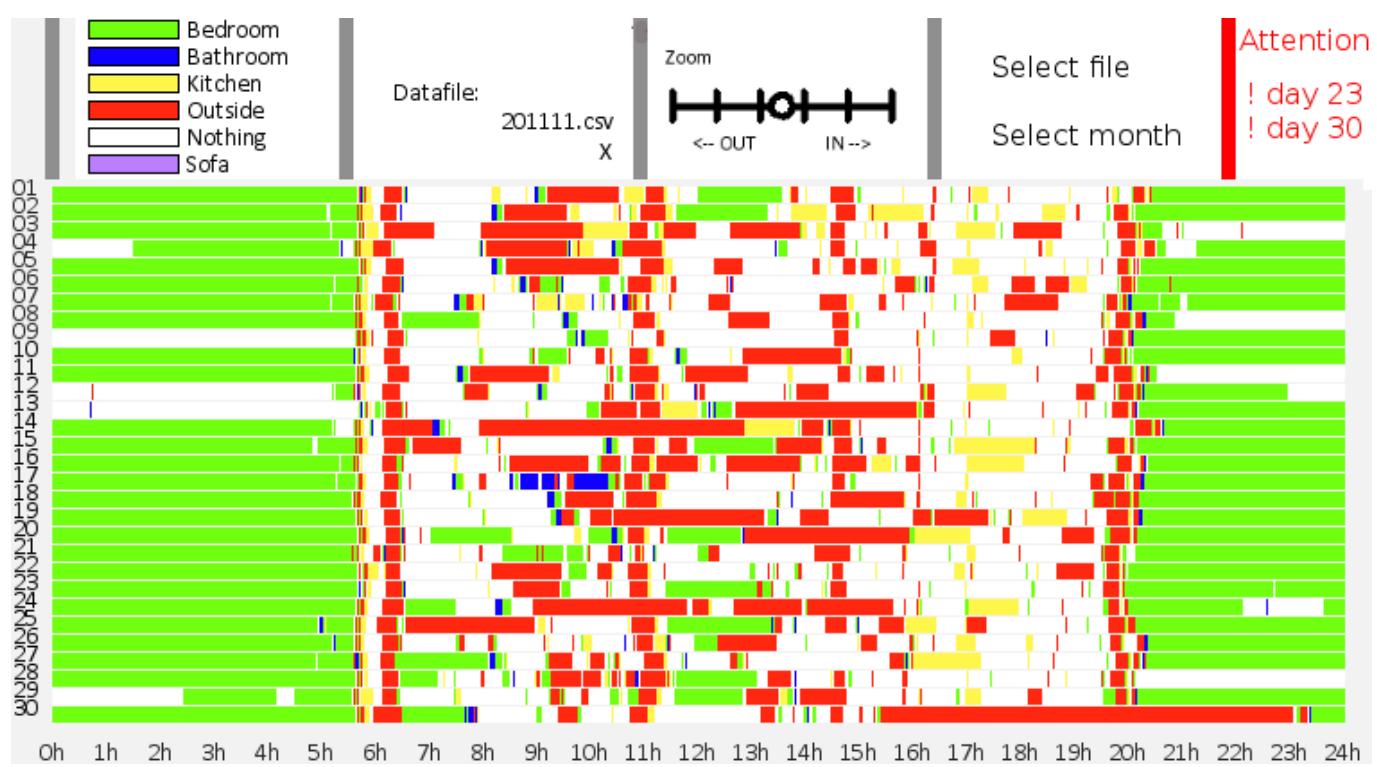

Fig. 4. A screenshot of the final application which was considered the most favoured visualization.

discussions indicated otherwise. In designing visualizations or identifying relevant changes, careful considerations have to be made regarding the inclusion and exclusion criteria of the participants. Different specialisms may lead to different results.

Besides determining that our currently presented results and conclusions are maintained in a larger sample, relevant deviations in the ADL and other activities need to be studied further for two reasons: (a) the identification of relevant deviations can be used for improving the system that generate alarms based on the sensor data, guiding the creation of a relevant set of rules or features, and (b) for effective support for the medical specialists it is important that a visualizing tool does not overload them with irrelevant information. As the data can reveal information about someone's habits and activity patterns, securing the data is important. The finding that the specialists were able to identify the person from the sensor data visualization strengthens this, because even when reports are anonymous, people's identity can be uncovered. Altogether, the developed visualizations helped an initial study to identifying relevant deviations in activities. The visualizations were also useful in explaining specialists why ADL deviations are relevant to identify, and to better understand how these should be visualized from the different perspectives of each medical specialism.

\section{ACKNOWLEDGMENTS}

The authors would like to thank the participants, Vivium Zorggroep Naarderheem, Sean Alizadeh and Margriet Pol. This work is supported by the Agentschap NL projects Health-lab and Design for Wellbeing and SIA project Smart Systems for Smart Services.

\section{REFERENCES}

[1] J. E. Cohen, "Human population: the next half century." Science, vol. 302, no. 5648, pp. 1172-5, 2003.

[2] E. Tapia, S. Intille, and K. Larson, "Activity recognition in the home using simple and ubiquitous sensors," Pervasive Computing, pp. 158175, 2004.
[3] S. Alizadeh, S. Bakkes, M. Kanis, M. Rijken, and B. Kröse, "Telemonitoring for assisted living residences: the medical specialists view." Med-E-Tel, 2011.

[4] S. Wang, M. Skubic, and Y. Zhu, "Activity density map dis-similarity comparison for eldercare monitoring," in Engineering in Medicine and Biology Society, 2009. EMBC 2009. Annual International Conference of the IEEE. IEEE, 2009, pp. 7232-7235.

[5] G. Virone, M. Alwan, S. Dalal, S. Kell, B. Turner, J. Stankovic, and R. Felder, "Behavioral patterns of older adults in assisted living," Information Technology in Biomedicine, IEEE Transactions on, vol. 12, no. 3, pp. 387-398, 2008.

[6] N. M. Boers, D. Chodos, J. Huang, P. Gburzynski, I. Nikolaidis, and E. Stroulia, "The smart condo: Visualizing independent living environments in a virtual world," in Pervasive Computing Technologies for Healthcare, 2009. PervasiveHealth 2009. 3rd International Conference on. IEEE, 2009, pp. 1-8.

[7] N. Gil, N. Hine, J. Arnott, J. Hanson, R. Curry, T. Amaral, and D. Osipovic, "Data visualisation and data mining technology for supporting care for older people," in Proceedings of the 9th international ACM SIGACCESS conference on Computers and accessibility. ACM, 2007, pp. 139-146.

[8] M. Mulvenna, W. Carswell, P. McCullagh, J. C. Augusto, H. Zheng, P. Jeffers, H. Wang, and S. Martin, "Visualization of data for ambient assisted living services," Communications Magazine, IEEE, vol. 49, no. 1, pp. 110-117, 2011.

[9] M. Rantz, M. Skubic, R. Koopman, G. Alexander, L. Phillips, K. Musterman, J. Back, M. Aud, C. Galambos, R. Guevara, and S. Miller, "Automated technology to speed recognition of signs of illness in older adults," Journal of Gerontological Nursing, vol. 38, no. 4, pp. $18-23,2012$.

[10] B. Kröse, M. Veenstra, S. Robben, and M. Kanis, "Living labs as educational tool for ambient intelligence," in Ambient Intelligence, ser. Lecture Notes in Computer Science, F. Paternò, B. de Ruyter, P. Markopoulos, C. Santoro, E. van Loenen, and K. Luyten, Eds., vol. 7683. Springer, 2012, pp. 356-363.

[11] D. Chang and K. Nesbitt, "Developing gestalt-based design guidelines for multi-sensory displays," in Proceedings of the 2005 NICTA-HCSNet Multimodal User Interaction Workshop-Volume 57. Australian Computer Society, Inc., 2006, pp. 9-16.

[12] C. G. Healey and J. T. Enns, "Attention and visual memory in visualization and computer graphics," Visualization and Computer Graphics, IEEE Transactions on, vol. 18, no. 7, pp. 1170-1188, 2012. 